\title{
Correlación entre las buenas prácticas de manufactura y el cumplimiento de los criterios microbiológicos en la fabricación de helados en Chile
}

\author{
Correlation between the good manufacturing practices \\ and compliance with microbiological criteria in \\ the manufacture of ice cream in Chile
}

\begin{abstract}
The Good Manufacturing Practices (GMP) is a basic tool to obtain safe foodstuffs for human consumption. The main goal of this research was to determine the percentage of compliance with GMP in ice cream factories and its correlation with the acceptance of microbiological criteria in samples of ice cream, according to the Sanitary Chilean Food Regulation. Inspectors from the Ministerial Regional Secretariat (SEREMI) of Health Nuble audited GMP in 40 ice cream factories and 435 microbial analyses from ice cream samples taken between the years 2005 and 2010 were analyzed. Results showed that $55.2 \%$ of samples failed in RAM (105 UFC/g) and coliform (102 UFC/g) and 4.6\% for Staphylococcus aureus (102 UFC/g) and 100\% for Salmonella spp. The biggest RAM was during summer with $60 \%$ rejection. Sixty seven $\%$ of inspections of GMP reached values above the minimum (70\% compliance). Facilities were the best item assessed (88.4\%), and training was the worst $(20.3 \%)$. The correlation between the results of audits of GMP and microbiological criteria allowed proving that with a percentage equal to or greater than $80 \%$ compliance with GMP would ensure the microbiological quality of ice cream, thereby decreasing the risk for consumer health.

Key words: Good Manufacturing Practices, microbiological quality, ice cream, food safety.
\end{abstract}

\section{INTRODUCCIÓN}

Las infecciones e intoxicaciones transmitidas por alimentos, constituyen un problema importante de salud pública y como consecuencia de esto tiene grandes repercusiones económicas en la industria alimentaria (1). Es por ello, que se trata de buscar nuevas y mejores métodos de diagnóstico como técnicas de biología molecular (PCR) e inmunológicas (ELISA) para poder obtener respuestas y así enfrentar de la mejor forma posible problemas que afectan la salud humana (2).

La calidad como resguardo de inocuidad, es que el alimento no cause daño a la salud de las personas que lo consumen, corresponde al nivel básico que debe satisfacer un producto alimenticio y es generalmente aquel controlado a nivel de estado o país para resguardar la salud pública de los ciudadanos (3). Para las personas susceptibles (niños, ancianos, mujeres embarazadas y personas enfermas), las enfermedades trasmitidas por alimentos (ETAs) pueden ser muy graves y dejar secuelas e incluso provocar la muerte (4).
José Miguel Bastías M. (1)

Marcela Cuadra H. (2)

Ociel Muñoz F. (3)

Roberto Quevedo L. (4)

(1) Departamento de Ingeniería en Alimentos, Universidad del Bío Bío, Chillán, Chile (2) Secretaria Regional Ministerial de Salud, Delegación Ñuble, Chillán, Chile. (3) Instituto de Ciencia y Tecnología en Alimentos, Universidad Austral de Chile, Valdivia, Chile. (4) Departamento de Ciencia y Tecnología de Alimentos, Universidad de los Lagos, Osorno, Chile.

Dirigir la correspondencia a: Dr. José Miguel Bastías Montes Departamento Ingeniería en Alimentos Universidad del Bío-Bío Casilla 447, Chillán, Chile Teléfono 56-42-463072 - 56-42-463073 Fax 56-42-463066

E-mail: jobastias@ubiobio.cl

Este trabajo fue recibido el 2 de Mayo de 2012, aceptado con modificaciones el 20 de Marzo de 2013 y aceptado para ser publicado el 5 de Mayo de 2013.

Las Buenas Prácticas de Manufactura (BPM) son una herramienta básica para la obtención de productos seguros para el consumo humano, que se centralizan en la higiene y en la forma de manipulación (5). La Organización Panamericana de la Salud ha definido las BPM, como el método moderno para el control de las enfermedades trasmitidas por alimentos a utilizar por parte de los gobiernos e industrias. Con la incorporación de esta herramienta, la industria sería el responsable primario de la inocuidad de los alimentos. (1)

De acuerdo al Reglamento Sanitario de los Alimentos de Chile (RSA), los establecimientos de producción, elaboración, preservación y envase de alimentos deben cumplir con las BPM, en forma sistematizada y auditable. La gestión de calidad de una empresa está basada en primer lugar en las BPM, que asimismo son el punto de partida para la implementación de otros sistemas de aseguramiento de calidad (5).

Este proceso, asegura tener bajo control la producción, ingreso de las materias primas, documentación, proceso de 
elaboración, almacenamiento, transporte y distribución de los alimentos. Por último las BPM son procedimientos de higiene y manipulación, que constituyen los requisitos básicos e indispensables para participar en el mercado (6).

De acuerdo a lo anterior, se planteó como objetivo determinar el porcentaje de cumplimiento de las Buenas Prácticas de Manufacturas (BPM) y su correlación con la aceptación de los criterios microbiológicos en las fábricas y muestras de helados de la provincia de Ñuble, Chile, de acuerdo a lo establecido en el Reglamento Sanitario de los Alimentos chilenos.

\section{MATERIALES Y MÉTODOS}

Se recopilaron y clasificaron los resultados microbiológicos de 435 muestras de helados provenientes de la provincia de Ñuble, entregadas por el laboratorio de análisis de alimentos de la Secretaria Regional Ministerial (SEREMI) de Salud Delegación Ñuble. El análisis microbiológico se determinó de acuerdo a las metodologías establecidas en el Manual de Análisis Bacteriológicos (BAM) (7). Los parámetros microbiológicos fueron los estipulados en el Reglamentos Sanitario de los alimentos (RSA), (recuento aerobios mesófilos (RAM), coliformes, Staphylococcus aureus y Salmonella spp) siguiendo los criterios microbiológicos que establece la legislación vigente (5).

Con la finalidad de estudiar si la estacionalidad climática afecta negativamente el recuento de aerobios mesófilos en las muestras de helados, se dividieron las muestras en dos grupos: las obtenidas durante los meses de Octubre a Marzo a las que corresponden muestras de primavera-verano y las obtenidas durante los meses de Abril a Septiembre muestras de otoño- invierno.

Paralelamente a lo anterior, se recopilaron y clasificaron los resultados de auditorías de Buenas Prácticas de Manufactura (BPM) aplicadas por fiscalizadores de la Unidad de Seguridad Alimentaria de la Delegación Ñuble de la SEREMI de Salud Bío Bío, a las mismas fabricas de helados muestreadas.

De acuerdo a lo establecido por el Ministerio de Salud Chileno (MINSAL), se considera que un establecimiento cumple con las BPM si reúne las siguientes condiciones: Sí cumple con el $100 \%$ de los cinco factores críticos, que son: autorización sanitaria de funcionamiento, abastecimiento de agua potable, manejo de residuos sólidos, disposición de residuos líquidos, servicios higiénicos de los manipuladores, y sí el porcentaje de cumplimiento de las auditorías de BPM es igual o superior al 70\%. La lista de chequeo aplicada, es la establecida por Ministerio de Salud de Chile que incluye los siguientes ítems: instalaciones, limpieza y sanitización, control de plagas, higiene del personal, capacitación, materias primas y procesos y productos terminados.

Para los análisis estadísticos, se utilizó el programa estadístico SPSS versión 13.0, realizando las pruebas de medidas de frecuencias, Chi- cuadrado, coeficiente de correlación de Pearson, matriz de correlación multivariado.

\section{RESULTADOS}

La tabla 1 muestra que el porcentaje de rechazo en las muestras de helados en los seis años de estudio para RAM fue siempre mayor que la de aceptación, excepto para el año 2008 y 2010. Se determinó que el año 2007 correspondió al año con menor aceptación ya que el $80 \%$ de las muestras de helados sobrepasaron los valores establecidos en el RSA. En cambio en los años 2008 y 2010 fueron significativamente mayores con un $62 \%$ de aceptación de acuerdo a lo estipulado en el RSA (5).

En la tabla 2 se observa el efecto de la estacionalidad respecto RAM en las muestras de helados analizados, los cuales presentaron diferencias estadísticamente significativas, es decir la época del año primavera - verano afecta la calidad microbiológica en cuanto a RAM, ya que 60,9\% de la muestras analizadas no cumplen con lo establecido en el RSA, en cambio en época otoño - invierno el porcentaje de no cumplimiento es significativamente menor alcanzando sólo un $34,8 \%$.

En cuanto al recuento de coliformes, se puede apreciar en la tabla 3 que existen diferencias estadísticamente significativas, o sea que en los distintos años estudiados la calidad microbiológica de los helados no presenta una tendencia a la mejora, eso se puede notar en la heterogeneidad de los resultados. El porcentaje de menor cumplimiento de la calidad para recuento de coliformes lo presentaron los años 2006 y 2007 con un $26 \%$ respecto a lo establecido en el RSA, a diferencia del $100 \%$ de cumplimento presentado el año 2009.

El menor porcentaje de cumplimiento en las muestra

\section{TABLA 1}

Porcentaje de aceptación en muestras de helados de acuerdo al recuento de aerobios mesófilos (RAM) conforme al Reglamento Sanitario de Alimentos.

AÑOS

\begin{tabular}{lcc} 
& \multicolumn{2}{c}{ Aceptación } \\
& $\mathrm{n} / \mathrm{N}$ & $\%$ \\
2005 & $5 / 13$ & 38, \\
2006 & $5 / 15$ & 33, \\
2007 & $3 / 15$ & 20, \\
2008 & $8 / 13$ & 61, \\
2009 & $3 / 7$ & 42, \\
2010 & $15 / 24$ & 62,
\end{tabular}

\section{CALIDAD MICROBIOLÓGICA}

$\%$

38,5

33,3

20,0

61,5

42,9

62,5
Rechazo

$\begin{array}{cc}\mathrm{n} / \mathrm{N} & \% \\ 8 / 13 & 61,5 \\ 10 / 15 & 66,7 \\ 12 / 15 & 80,0 \\ 5 / 13 & 38,5 \\ 4 / 7 & 57,1 \\ 9 / 24 & 37,5\end{array}$


de helados respecto a Staphylococcus aureus de acuerdo al RSA (tabla 4) fue el año 2006 (86,7\%), presentado diferencia estadísticamente significativa con los años 2007, 2009 y 2010 donde el porcentaje de aceptación alcanzó el 100\%.

\section{TABLA 2}

Porcentaje de aceptación en muestras de helados respecto a la estacionalidad de acuerdo al recuento de aerobios mesófilos (RAM) conforme al Reglamento Sanitario de Alimentos.

\begin{tabular}{|c|c|c|c|c|}
\hline \multirow[t]{3}{*}{ Estacionalidad } & \multicolumn{4}{|c|}{ CALIDAD MICROBIOLÓGICA } \\
\hline & \multicolumn{2}{|c|}{ Rechazo } & \multicolumn{2}{|c|}{ Aceptación } \\
\hline & $\mathrm{n} / \mathrm{N}$ & $\%$ & $\mathrm{n} / \mathrm{N}$ & $\%$ \\
\hline Primavera- Verano & $39 / 64$ & 60,9 & $25 / 64$ & 39,1 \\
\hline Otoño-Invierno & $8 / 23$ & 34,8 & $15 / 23$ & 65,2 \\
\hline $\begin{array}{l}p=0,031 \\
n: \text { Lotes de muestras de helad } \\
\text { N: Total de lotes muestreados }\end{array}$ & des. & & & \\
\hline
\end{tabular}

TABLA 3

Porcentaje de aceptación en muestras de helados de acuerdo al recuento de coliformes conforme al Reglamento Sanitario de Alimentos.

\begin{tabular}{|c|c|c|c|c|}
\hline \multirow[t]{3}{*}{ AÑOS } & \multicolumn{4}{|c|}{ CALIDAD MICROBIOLÓGICA } \\
\hline & \multicolumn{2}{|c|}{ Rechazo } & \multicolumn{2}{|c|}{ Aceptación } \\
\hline & $\mathrm{n} / \mathrm{N}$ & $\%$ & $\mathrm{n} / \mathrm{N}$ & $\%$ \\
\hline 2005 & $4 / 13$ & 30,8 & $9 / 13$ & 69,2 \\
\hline 2006 & $11 / 15$ & 73,3 & $4 / 15$ & 26,7 \\
\hline 2007 & $11 / 15$ & 73,3 & $4 / 15$ & 26,7 \\
\hline 2008 & $6 / 14$ & 42,9 & $8 / 14$ & 57,1 \\
\hline 2009 & $0 / 7$ & 0,0 & $7 / 7$ & 100,0 \\
\hline 2010 & $16 / 23$ & 69,6 & $7 / 23$ & 30,4 \\
\hline $\begin{array}{l}\mathrm{b}=0,002 \\
\mathrm{n}: \text { Lotes de mu } \\
\mathrm{N}: \text { Total de lot }\end{array}$ & & & & \\
\hline
\end{tabular}

TABLA 4

Porcentaje de aceptación en muestras de helados de acuerdo a pesquisa de Staphylococcus aureus conforme al Reglamento Sanitario de los Alimentos.

\section{AÑOS}

$\begin{array}{cc} & \mathrm{n} / \mathrm{N} \\ 2005 & \\ 2006 & 1 / 13 \\ 2007 & 2 / 15 \\ 2008 & 0 / 15 \\ 2009 & 1 / 14 \\ 2010 & 0 / 7 \\ & 0 / 23\end{array}$

\section{CALIDAD MICROBIOLÓGICA}

Rechazo

$\%$

6,8

13,3

0,0

7,1

0,0

0,0
Aceptación

$\mathrm{n} / \mathrm{N} \quad \%$

$12 / 13$
$13 / 15$
$15 / 15$
$13 / 14$
$7 / 7$
$23 / 23$

92,3

86,7

100,0

92,9

100,0

100,0 
En la tabla 5 se puede apreciar que el 100\% de las muestras de helados analizadas durante los seis años de estudios cumple con la calidad microbiológica para Salmonella spp de acuerdo a lo establecido en el RSA.

Al evaluar el grado de cumplimiento de las BPM en las 40 fábricas de helados estudiadas (figura 1), se encontró que el $67 \%$ de ellas $(n=27)$ arrojaron un valor superior a $70 \%$, que corresponde al mínimo de aceptación en las Auditorias de las BMP establecido por el MINSAL. Del total de fábricas un $20 \%$ $(n=8)$ supera el $91 \%$ de cumplimiento, en cambio solo el $33 \%$ $(n=13)$ presentó un porcentaje inferior a lo establecido por el MINSAL para el cumplimiento de las BPM.

Como se observa en figura 2 podemos destacar que los ítems limpieza y sanitización, control de plagas, capacitación y materias primas estaban bajo el $70 \%$ de cumplimiento en promedio, a diferencia de los ítems instalaciones, higiene del personal y proceso y producto terminado que sobrepasan el porcentaje de cumplimiento para BPM.

En relación al grado de correlación de los diferentes ítems de las BPM considerados por el MINSAL, estos fueron indicados con un asterisco $\left(^{*}\right)$ ubicado en el interior de los rectángulos que se presentan en figura 3. Los ítems que presentaron la mejor correlación fueron limpieza y sanitización con capacitación con un valor de $r=0,6004$, seguido de higiene del personal con capacitación con $r=$ 0.541 , en cambio los ítems que presentaron el menor grado de correlación fueron capacitación con materia prima con un $r=1,383$.

Respecto a la correlación que existe entre el porcentaje de cumplimiento de BPM y la calidad microbiológica de los

\section{TABLA 5}

Porcentaje de aceptación de Salmonella spp en muestras de helados conforme al Reglamento Sanitario de los Alimentos.

\begin{tabular}{|c|c|c|}
\hline \multirow[t]{3}{*}{ AÑOS } & \multicolumn{2}{|c|}{ CALIDAD MICROBIOLÓGICA } \\
\hline & \multicolumn{2}{|c|}{ Ausencia de Salmonella spp } \\
\hline & $\mathrm{n}$ & $\%$ \\
\hline 2005 & 65 & 100,0 \\
\hline 2006 & 75 & 100,0 \\
\hline 2007 & 75 & 100,0 \\
\hline 2008 & 70 & 100,0 \\
\hline 2009 & 35 & 100,0 \\
\hline 2010 & 115 & 100,0 \\
\hline Total & 435 & 100,0 \\
\hline
\end{tabular}

\section{FIGURA 1}

Frecuencias absolutas de porcentaje de cumplimiento de las buenas prácticas de manufactura (BPM) de fábricas de helados.

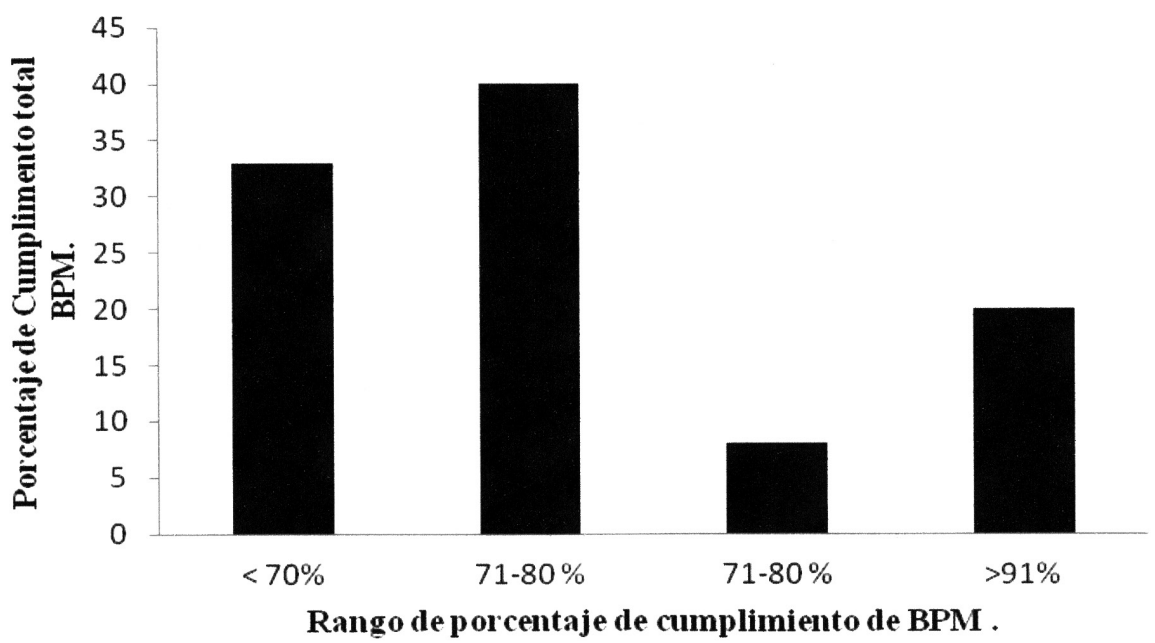




\section{FIGURA 2}

Porcentaje de cumplimiento de los diferentes ítems evaluados en las auditorías de buenas prácticas de manufacturas (BPM) aplicadas a fábricas de helados.

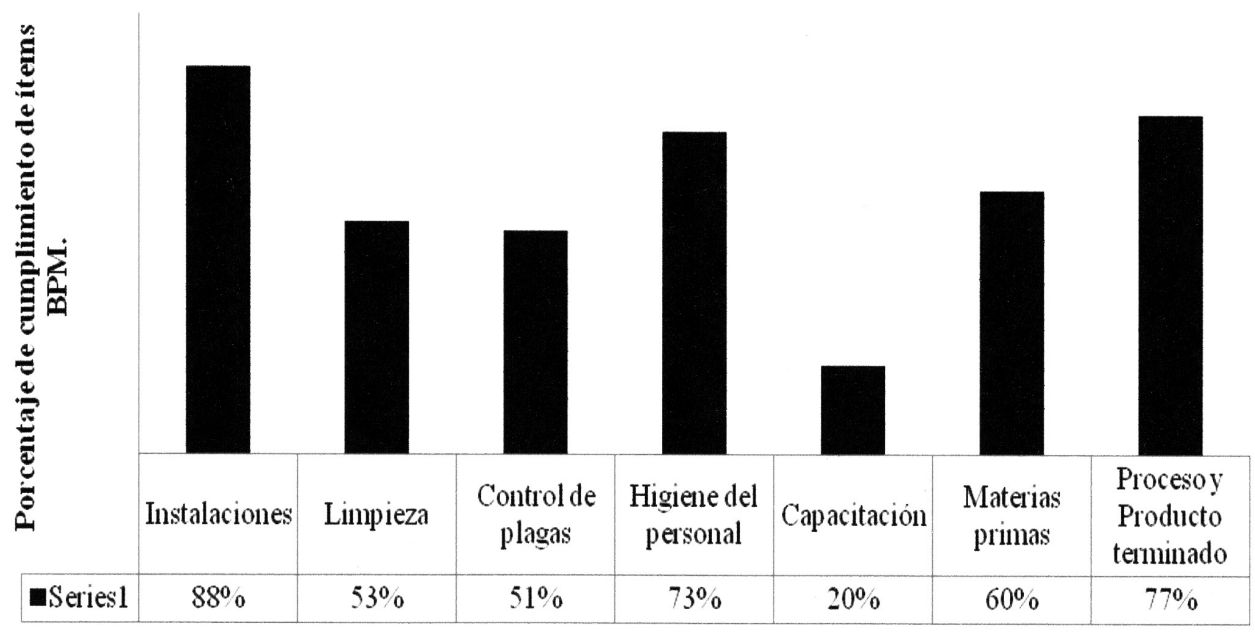

\section{FIGURA 3}

Mátriz de correlación multivariada entre los diferentes ítems de las buenas prácticas de manufacturas (BPM) aplicadas a fábricas de helados.

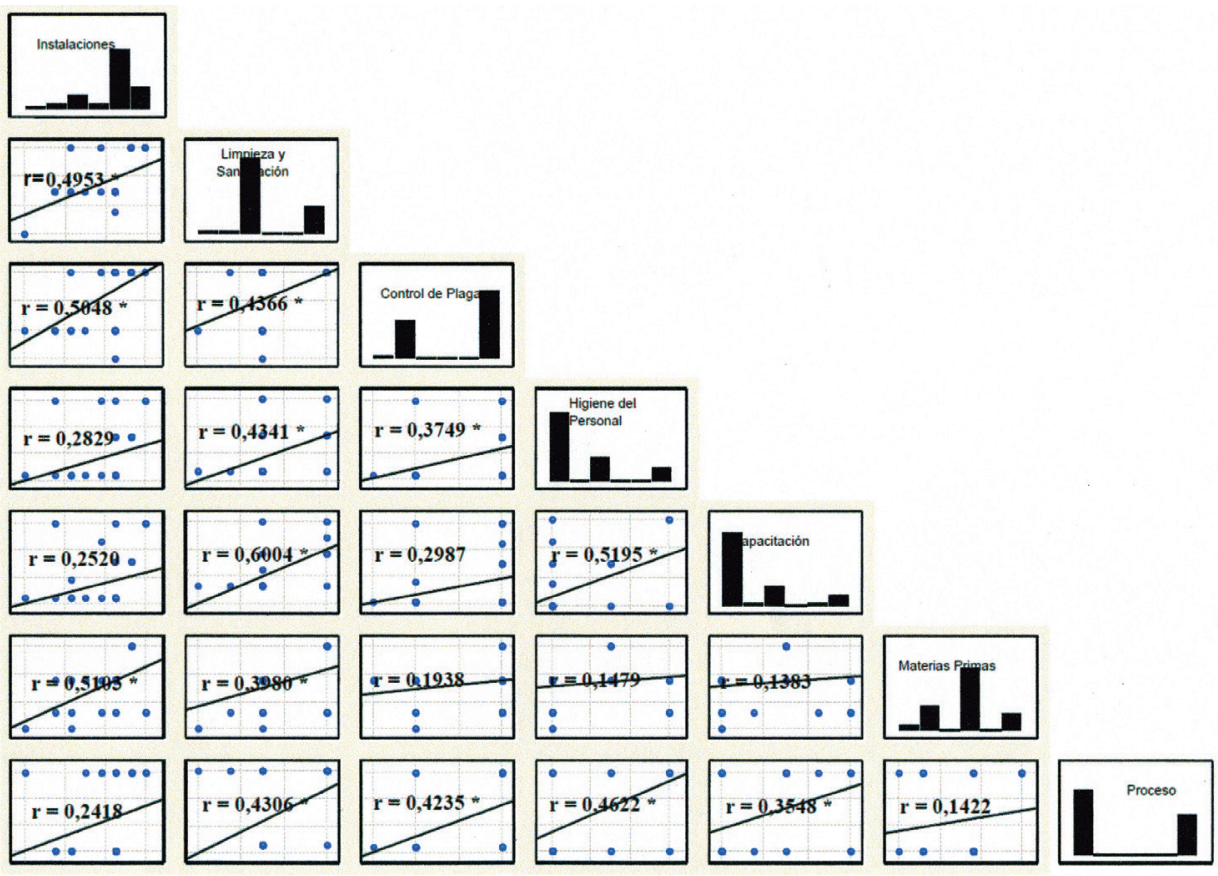


helados elaborados en la provincia de Ñuble (figura 4), se pudo determinar que con un cumplimiento de un $79,13 \%$ de BPM se aseguraría la calidad microbiológica de los helados de acuerdo a lo establecido por el RSA.

\section{DISCUSIÓN}

El nivel de rechazo en RAM de las muestras de helados en el presente estudio es menores que otras investigaciones similares, sólo los resultados del 2006 se asemejan a los obtenidos en México (8), donde el porcentaje de rechazo en RAM fue de un $75 \%$, en cambios estudios realizado en helados en Argentina (9) presentaron valores superiores de rechazo (95\%), incluso en helados analizados en Venezuela se llegó al $100 \%$ de rechazo (10). Los altos niveles de rechazo en RAM pueden ser multivariados ya que podrían estar asociados a una deficiente calidad en la materia prima, problemas de limpieza, sanitización, deferencia durante el procesamiento, incluso en el transporte y almacenamiento $(11,12)$, en todo caso se debe tener presente que un RAM bajo no asegura que un alimento esté exento de patógenos o sus toxinas (13). En relación a un mayor porcentaje de rechazo en RAM en temporada estival se puede asociar a un efecto de temperaturas ambientales superiores, lo cual también fue confirmado por resultados similares obtenido en Libia (14).

Referente a los coliformes en las muestras de helados, otros estudios han logrado demostrar niveles superiores de rechazos, como es el caso de dos investigaciones independientes realizadas en Venezuela donde el 100\% de las muestras de helados presentaron valores por sobre el límite de aceptación $(10,15)$, hallazgo similar se encontró en muestras de helados en Argentina con un $90 \%$ de rechazo (9). En cambio resultados muy próximo a los obtenidos durante los años 2006 y 2007 fueron informado en un trabajo realizado en Libia (13) y otro en Venezuela (16), a diferencia de un estudio realizado en
México (8) donde los valores de rechazo son similares a los obtenidos en el 2008. Resultados de recuentos de coliformes encontrados en muestras de helados en Turquía (3) presentaron valores de rechazo de un 25\% similares al año 2005 del presente estudio. La heterogeneidad de estos resultados discutidos con anterioridad, puede estar relacionado a un problema de manipulación durante la elaboración y por ende se puede asociar a una deficiente capacitación del personal.

Staphylococcus aureus son microorganismos fácilmente destruidos por tratamientos térmicos con altas temperaturas y por agentes sanitizantes, por lo cual la presencia de esta bacteria o sus toxinas en alimentos procesados o en equipos, generalmente indicaría falta de sanitización o contaminación cruzada (14). De los seis años de análisis en las muestra de helados en la presente investigación, sólo tres arrojaron resultados fuera de norma cuyo valores no superaron el $13 \%$. Al revisar estudio efectuados en Venezuela se comprobó que obtuvieron valores muy superiores de rechazo entre un $65 \%$ y $100 \%(16,10)$, en cambio otras dos investigaciones efectuadas en el mismo país no pesquisaron Staphylococcus aureus, confirmando con ello una buena calidad en el proceso de elaboración de helados $(15,17)$. Las muestras de helados que presentan Staphylococcus aureus podrían estar representado un riesgo para la salud de los consumidores, particularmente para la población infantil y personas inmunodeprimidos y de la tercera edad (14).

Resultados obtenidos en esta investigación para SalmoneIla spp, fueron similares a estudios realizados en Turquía $(3,17)$ y Venezuela $(16,18)$, en cambio investigaciones realizadas en muestras de helados en Libia (14) y Argentina (10) encontraron presencia de Salmonella spp, con el consiguiente riesgo para el consumidos, indicando una deficiente manipulación de los alimentos en el proceso de elaboración de los helados, producto de contaminación cruzada.

\section{FIGURA 4}

Correlación entre grado de clumpimiento de los criterios microbiológicos de helados y el promedio de los resultados de las auditorías de BPM realizadas a las fábricas de helados.

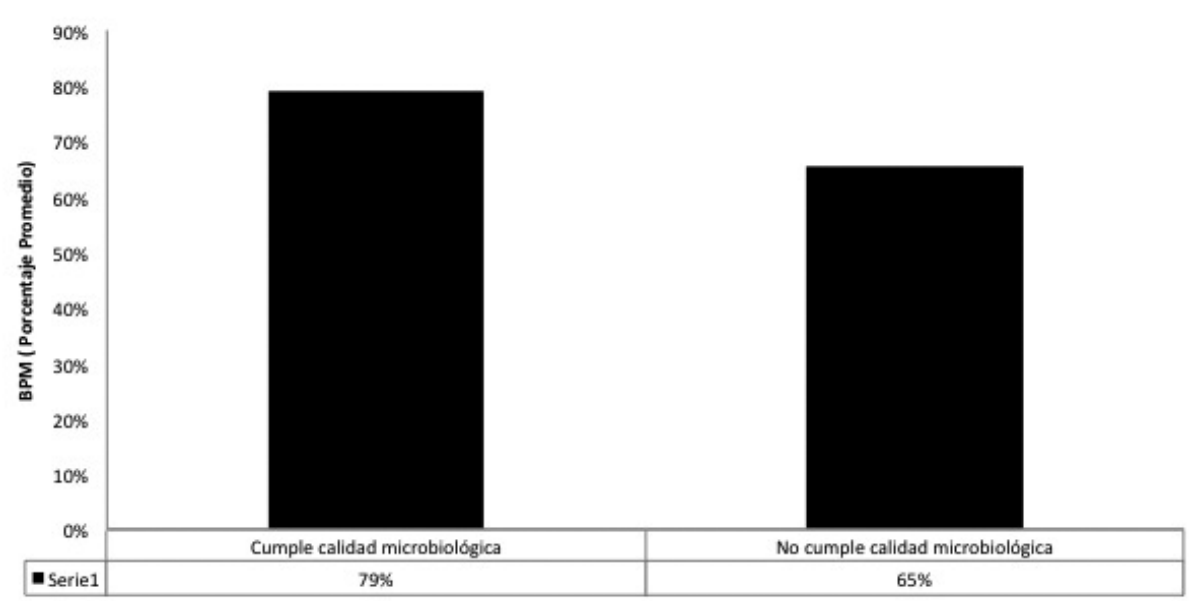


Una de las principales causas de contaminación en la elaboración de alimentos es la inexistencia de adecuadas técnicas de limpieza y desinfección en las áreas de procesos, algunas veces por desconocimiento por parte de manipuladores (19). Asegurar la calidad de los alimentos implica tener implementado un plan de limpieza y desinfección que coadyuve, lo que en conjunto con las BPM reducen al mínimo el peligro de contaminación y aseguraría la inocuidad de los productos (1, $5,20)$. Los resultados encontrados en la presente investigación respecto a las BPM permitieron comprobar que el nivel de cumplimiento es superior al informado en otros estudios. Auditoría en una empresa de helados del municipio de Soacha en Venezuela informaron que el nivel de cumplimiento en las BPM sólo alcanzó promedio de $40 \%$ (18), en cambio una fábrica de cecinas auditada en Colombia arrojó un $68 \%$ de cumplimiento en BPM (20), a diferencia de la evaluación de los servicios de alimentacion en instituciones infantiles en el mismo país donde se encontró un porcentaje de cumplimiento de BPM de sólo 20\% (19).

Estudios realizados en Costa Rica respecto a la implementación de las BPM en una industria panificadora, arrojó valores de cumplimiento en el ítem instalaciones del 51\%, limpieza y sanitización $56 \%$, higiene del personal $68 \%$, control de plagas $44 \%$, capacitación $68 \%$, materias primas $38 \%$ y proceso y producto terminado $59 \%$, con un porcentaje promedio en BPM de 55\% (21). Si comparamos estos resultados y los de la presente investigación los ítems limpieza, control de plagas y capacitación presentaron menores valores de cumplimiento, sin embargo el promedio general de BPM fue muy superior, determinando que sobre un promedio de $80 \%$ de cumplimiento en BPM se aseguraría la calidad microbiológica en la elaboración de los helados y por ello se sugiere que el porcentaje de cumplimiento de un $70 \%$ en las auditorías de BPM debería subir a un $80 \%$.

\section{CONCLUSIONES}

La calidad microbiológica de los helados elaborados por las fábricas de la provincia de Ñuble en Chile respecto a RAM y coliformes fueron deficientes asociado principalmente a una baja capacitación del personal, respecto a Staphylococcus aureus y Salmonella spp presentaron una buena calidad. Referente a la correlación entre BPM y la calidad microbiológica se estableció que con un porcentaje de cumplimiento de $80 \%$ en BPM se puede asegurar la calidad e inocuidad microbiológica en la elaboración de los helados.

\section{RESUMEN}

Las Buenas Prácticas de Manufacturas (BPM) son una herramienta básica para obtener un producto alimenticio seguro para consumo humano. El objetivo de este estudio fue determinar el porcentaje de cumplimiento de BPM y su correlación con la aceptación de criterios microbiológicos en fábricas y muestras de helados de Ñuble, Chile, de acuerdo al Reglamento Sanitario de Alimentos chilenos. Se analizaron auditorías de BPM aplicadas por fiscalizadores de Secretaría Regional Ministerial (SEREMI) de Salud Ñuble, a 40 fábricas de helados y los resultados microbiológicos de 435 muestras de helados entre los años 2005 y 2010. Se determinó que el $55,2 \%$ de las muestras no cumplieron para RAM (105 UFC/g) y coliformes (102 UFC/g) y 4,6\% para Staphylococcus aureus (102 UFC/g), en cambio el $100 \%$ cumplieron para Salmonella spp. Los mayores RAM fueron en verano con $60 \%$ de rechazo. El $67 \%$ de auditorías de BPM alcanzaron valores superiores al mínimo (70\% de cumplimiento), las instalaciones fue el ítem mejor evaluado (88,4\%), y capacitación del personal el peor (20,3\%). La correlación entre los resultados de auditorías de BPM y criterios microbiológicos permitió comprobar que con un porcentaje igual o superior al $80 \%$ de cumplimiento de BPM se aseguraría la calidad microbiológica de helados, disminuyendo con ello el riesgo en la salud del consumidor.

Palabras clave: Buenas Prácticas de Manufacturas, calidad microbiológica, helados, seguridad alimentaria.

\section{BIBLIOGRAFÍA}

1. Organización Mundial de la Salud (OMS). Inocuidad de los Alimentos. Informe de Secretaria. Consejo Ejecutivo $108^{a}$ reunión, abril, 2001. Documento pdf EB 108/7; 1.

2. Mossel D, Moreno B y Struijk. C. Microbiología de los Alimentos, Editorial Acribia; 2006.

3. Kanbakan U, Ayar A. Determination of microbiological contamination sources during ice cream production in Denizli, Turkey. Food Control. 2004; 15: 463-70.

4. Kuplulu O, Sarimehmetog/u B.Isolation and identification of Brucella spp. in ice cream. Food Control. 2004; 15: 511-14.

5. Ministerio de Salud de Chile. Reglamento Sanitario de los Alimentos chilenos D.S. 977, 2010, Ediciones Lexnova. 2010.

6. Puig J., Durán F. Ingeniería, Autocontrol y Auditoría de Higiene en Industria Alimentaria. A. Madrid Vicente Ediciones, Ediciones Mundi-Prensa; 2002.

7. Manual Analítico Bacteriológico, $8{ }^{a}$ Edición, Revisión A, 1998.

8. Landanery B, Rostro M. Evaluación microbiológica de helados en la zona dorada de Tampico. México. Spectro. 2008; 10 (3): 14-9.

9. Pernía M, Mendoza C. Calidad microbiológica de helados blandos de mantecado comercializados en la ciudad de Maracay estado Aragua. Venezuela. Rev Salud Pública Nutr. 2007; 3; 42-61.

10. Vizcaya T, González F, Gutiérrez O. Riesgo epidemiológico por helados no industriales en Barquisimeto, Venezuela, 2008. Comunidad y Salud, 2009, 7 (2): 1-9.

11. Jay J. Microbiología moderna de los alimentos. Editorial Acribia SA. Zaragoza. España. 2002.

12. Figueroa G. Estrategias para Prevenir la Contaminación Microbiana: Patógenos Alimentarios Emergentes. Rev Chil Nutr. 2000;27(3): 332- 9.

13. Watson D. Revisiones sobre Ciencia y tecnología de los Alimentos, (1) Higiene y Seguridad Alimentaria. Zaragoza. Editorial ACRIBIA, S.A. 1998

14. El-Sharef N, Ghenghesh K, Abognah Y, Gnan S, Rahouma A. Bacteriological quality of ice cream in Tripoli-Libya. Food Control. 2006, 17; 637-41.

15. Rosales Y, Díaz C. Evaluación de la calidad microbiológica de helados caseros en Mérida Venezuela. Rev Salud Pública Nutr. 2006; (7); 54-62.

16. Szwarcbort L; García C. Calidad microbiológica de los helados de crema elaborados en Caracas, Venezuela. Nutrición. 1989; 39: 46-56.

17. Kanbakan U, Ayar A. Determination of microbiological contamination sources during ice cream production in Denizli, Turkey. Food Control. 2004, 15; 463-70.

18. Ávila V, Silva M. Evaluación de la calidad microbiológica de los helados en una empresa del municipio de Soacha y su impacto a nivel local. Venezuela. Rev Salud Pública Nutr. 2008, 12; 45-51.

19. Vásquez de la plata G, Gómez de Avellaneda E, Gamboa E. 
Condiciones Higiénico sanitarias de los servicios de Alimentación en instituciones infantiles del instituto colombiano de bienestar familiar de Bucaramanga, Colombia. Rev Cubana Alimentación Nutr. 2007, 17; 23-33.

20. Hincapié, M. Implementación de las buenas prácticas de manufactura (BPM) en la microempresa el Chorizo Paisa en la ciudad de Pereira. Colombia. Rev Salud Pública Nutr. 2007, 71-5.

21. Smitter A. Evaluación del Grado de Avance y Propuesta de Implementación de un Programa de Buenas Prácticas de Manufactura, en la Industria Alimenticia Copeyana S.A. Rev Cubana Aliment Nutr. 2004, 12(1): 17-23. 